\title{
Beam measurement of the high frequency impedance sources with long bunches in the CERN Super Proton Synchrotron
}

\author{
A. Lasheen, ${ }^{1,2, *}$ T. Argyropoulos, ${ }^{1}$ T. Bohl, ${ }^{1}$ J. F. Esteban Müller, ${ }^{1}$ \\ H. Timko, ${ }^{1}$ and E. Shaposhnikova ${ }^{1}$ \\ ${ }^{1}$ CERN, CH-1211 Geneva, Switzerland \\ ${ }^{2}$ Université Paris-Sud, 91400 Orsay, France
}

(Received 20 June 2017; published 23 March 2018)

\begin{abstract}
Microwave instability in the Super Proton Synchrotron (SPS) at CERN is one of the main limitations to reach the requirements for the High Luminosity-LHC project (increased beam intensity by a factor 2). To identify the impedance source responsible of the instability, beam measurements were carried out to probe the SPS impedance. The method presented in this paper relies on measurements of the unstable spectra of single bunches, injected in the SPS with the rf voltage switched off. The modulation of the bunch profile gives information about the main impedance sources driving microwave instability, and is compared to particle simulations using the SPS impedance model to identify the most important contributions. This allowed us to identify the vacuum flanges as the main impedance source for microwave instability in the SPS, and to evaluate possible missing impedance sources.
\end{abstract}

DOI: 10.1103/PhysRevAccelBeams.21.034401

\section{INTRODUCTION}

Longitudinal instabilities in the Super Proton Synchrotron (SPS) at CERN are a major limitation for future projects, including the High Luminosity LHC (HL-LHC) [1] and the Advanced Proton Driven Plasma Wakefield Acceleration Experiment (AWAKE) [2]. A beam can become unstable due to its interaction with surroundings, whose effect on the beam can be described by a coupling impedance $\mathcal{Z}$. This limitation is studied in the frame of the LHC Injector Upgrade project (LIU) [3] to evaluate the most critical impedance sources and possible cures.

One of the instabilities which are critical in the SPS is the "microwave instability" that manifests in the SPS as a fast, uncontrolled longitudinal emittance blow-up above a certain intensity threshold, as shown in Fig. 1. This instability is driven by high frequency impedance sources for which:

$$
f_{r} \tau_{L} \gg 1
$$

where $f_{r}$ is the resonant frequency of the impedance and $\tau_{L}$ is the full bunch length ( $4 \sigma_{\mathrm{rms}}$ is the case of a Gaussian bunch profile). The $200 \mathrm{MHz}$ traveling wave cavities (TWC) is the main rf system in the SPS used for acceleration, so a typical

*alexandre.lasheen@cern.ch

Published by the American Physical Society under the terms of the Creative Commons Attribution 4.0 International license. Further distribution of this work must maintain attribution to the author(s) and the published article's title, journal citation, and DOI. bunch length in the SPS is $\tau_{L} \approx(1.5-3.5)$ ns. Then microwave instability could be driven by impedance sources with a resonant frequency above $f_{r}>1 \mathrm{GHz}$. A fourth-harmonic rf system is available in the SPS and used in bunchshortening mode to stabilize the LHC beam (by increased Landau damping). However, using this second rf system cannot cure completely this kind of instability $[4,5]$ which remains a significant limitation for both LHC and AWAKE beams.

A survey of many SPS elements was done and their impedances were evaluated from electromagnetic simulations and measurements. It now includes contributions from most of the expected sources, such as the TWC and their high-order modes (HOM), the injection/extraction kickers, the vacuum flanges with various geometries that can be grouped depending on their location: near the focusing quadrupole magnets QF or the defocusing quadrupole magnets QD (a vacuum flange is often paired with an unshielded bellow and the overall structure will be referred to as vacuum flanges for the rest of the paper), the pumping ports, the longitudinal space charge [6] and many more smaller sources $[7,8]$. The present SPS impedance model is shown in Fig. 2 and it is used in beam dynamics simulations for studies of instabilities.

The beam itself was also used to probe the machine impedance in various dedicated measurements that are valuable to build the impedance model. The method presented in this paper consists of measuring the density modulation of long bunches by high frequency impedances with the rf voltage switched off. This method was used in the past to identify the main impedance sources responsible for microwave instability on the SPS flat bottom. They 


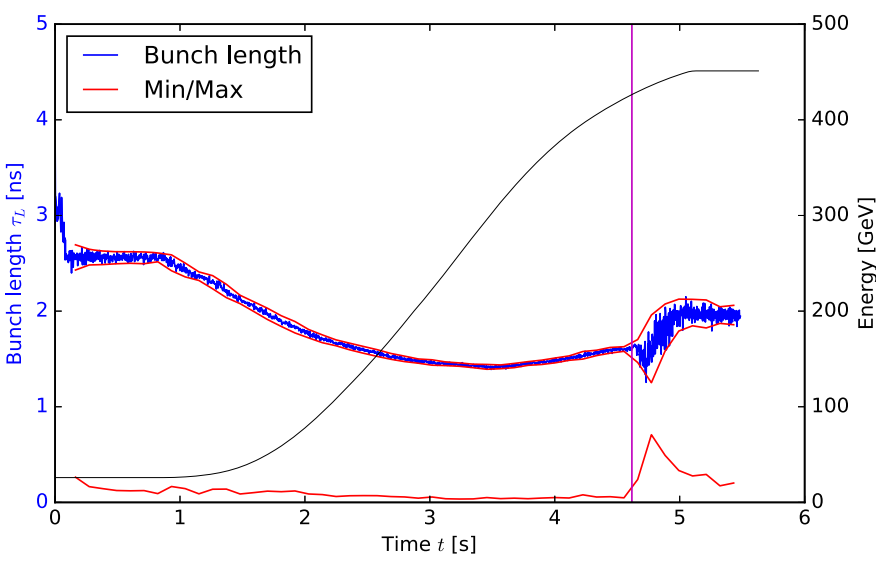

(a) $N_{b} \approx 1.4 \times 10^{11} \mathrm{ppb}$

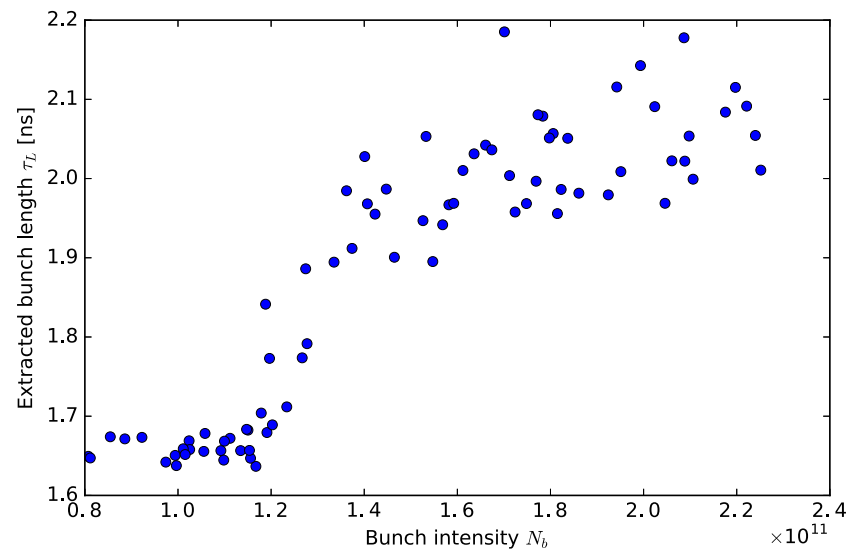

(b) Several acquisitions

FIG. 1. Longitudinal instability of a single bunch along the acceleration ramp in double rf operation: (a) Example of bunch length during the ramp the starting time of the instability is the magenta vertical line). The red line on the bottom shows the full amplitude (Max-Min) of bunch length oscillations. (b) The bunch length at the end of the cycle as a function of the bunch intensity $N_{b}$ for the same injected longitudinal emittance $\left(\varepsilon_{L} \approx 0.25 \mathrm{eVs}\right)$.

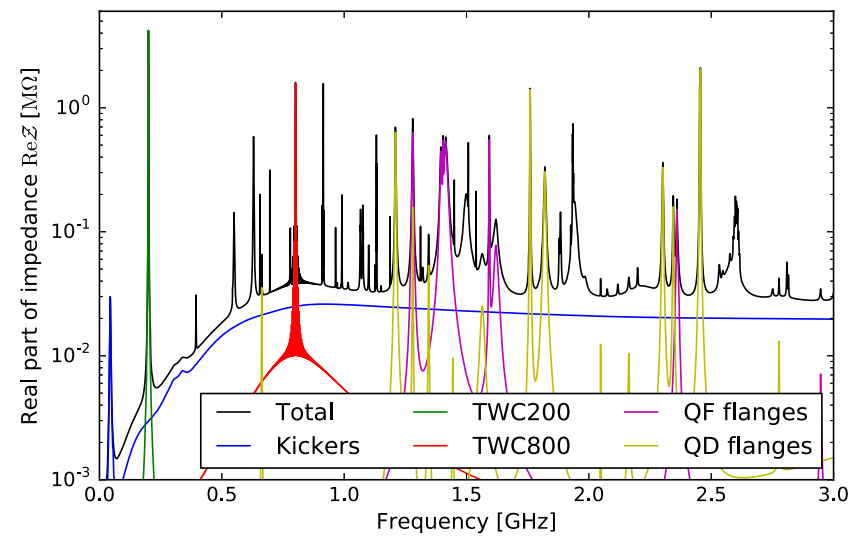

FIG. 2. The present SPS longitudinal impedance model (resistive part $\operatorname{Re} \mathcal{Z}$ ): the total impedance (blue) together with the most important subsets (various colors).

were the $\sim 800$ inter-magnet pumping ports [9] which were shielded during the 2000-2001 shutdown, allowing the SPS to reach a higher beam intensity [10]. More recently, investigations were done to identify the new impedance sources limiting the future projects.

In this paper, we will first describe the high frequency modulation of long bunches with rf off. Next, measurement results are presented (for two different SPS optics configurations) which allowed the most important impedance contributions to be identified. Finally, the present SPS impedance model is evaluated by comparison of macroparticle simulations with measurements.

\section{MICROWAVE INSTABILITY WITH RF OFF}

Measurements of the high frequency bunch profile were done on the SPS flat bottom (momentum $p=26 \mathrm{GeV} / \mathrm{c}$ ) with the rf voltage switched off. An example of a measured bunch profile modulation by high frequency impedance sources is shown in Fig. 3. To achieve this result, a long bunch was required to identify precisely the resonant frequency of the impedance source. Moreover, a small momentum spread $\Delta p_{m} / p$ was necessary for the debunching to be slow in comparison with the instability. The evolution of the bunch length $\tau_{L}$ during debunching with $\mathrm{rf}$ off is given by [11]:

$$
\tau_{L}(t)=\tau_{L 0}\left[1+\left(\frac{t}{t_{d}}\right)^{2}\right]^{\frac{1}{2}},
$$

where $\tau_{L 0}$ is the initial bunch length $t_{d}$ is the debunching time corresponding to:

$$
\frac{1}{t_{d}^{2}}=\left(\frac{2|\eta|}{\tau_{L 0}} \frac{\Delta p_{m}}{p}\right)^{2}+\left(\frac{6 N_{b} q^{2}|\eta| \operatorname{Im} \mathcal{Z} / n}{\pi E \tau_{L}}\right)^{2},
$$

where $\eta=\gamma_{t}^{-2}-\gamma^{-2}$ is the slippage factor $\left(\gamma_{t}\right.$ is the transition Lorenz factor, the SPS is above transition energy for all measurements presented below), $\Delta p_{m} / p$ is the maximum momentum spread of the bunch, $N_{b}$ is the bunch intensity, $q$ is the particle charge, $\operatorname{Im} \mathcal{Z} / n$ is the reactive impedance of the machine $\left(n=f / f_{\text {rev }}, f\right.$ is the frequency and $f_{\text {rev }}$ is the revolution frequency) and $E$ is the total beam energy. To minimize the debunching, a small momentum spread is required. Note that the dependence on $\operatorname{Im} \mathcal{Z} / n$ of the debunching time with $\mathrm{rf}$ off can be used to evaluate the reactive part of the machine impedance [11].

To get a convenient bunch distribution, the rf voltage was adiabatically decreased before extraction in the SPS injector (PS), which gave a bunch length of $\tau_{L} \approx(25-30) \mathrm{ns}$. This is more than ten times longer than the usual bunch length in the SPS $\tau_{L} \approx(1-3)$ ns. In this case, the frequency range of the stable bunch spectrum at injection is low $(f<100 \mathrm{MHz})$ as shown in Fig. 3(d). Therefore, all the 


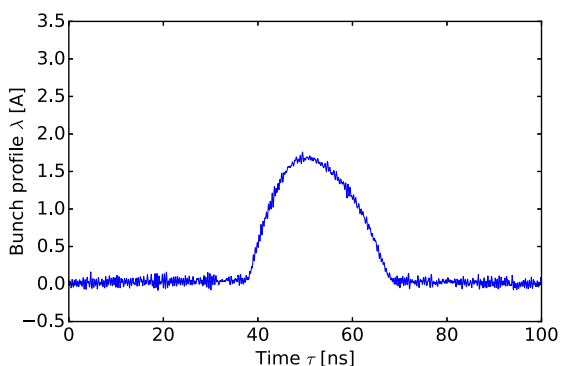

(a) Profile at $t=0 \mathrm{~ms}$

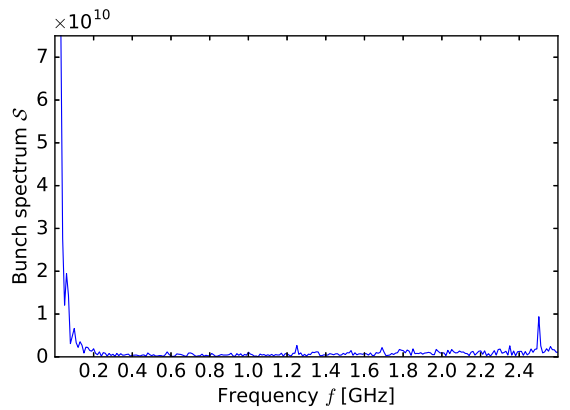

(d) Spectrum at $t=0 \mathrm{~ms}$

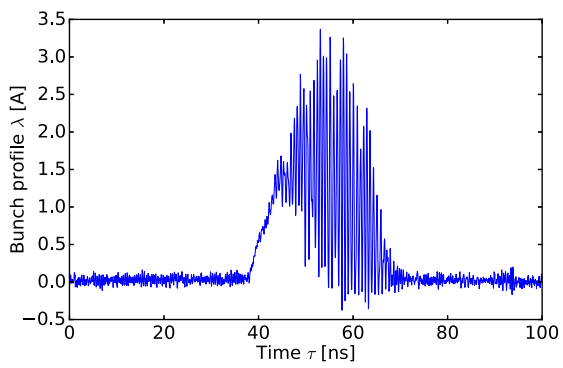

(b) Profile at $t=6.23 \mathrm{~ms}$

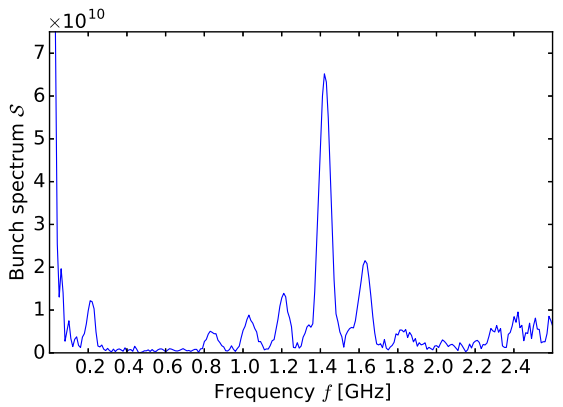

(e) Spectrum at $t=6.23 \mathrm{~ms}$

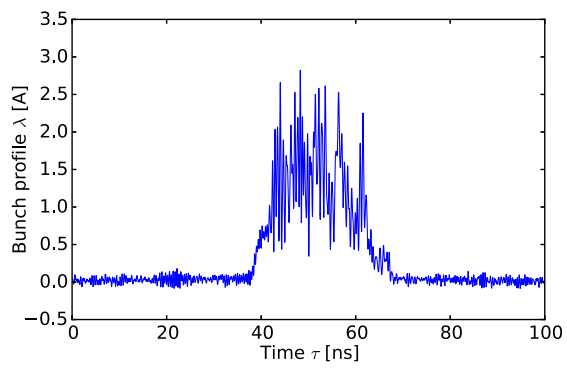

(c) Profile at $t=9.24 \mathrm{~ms}$

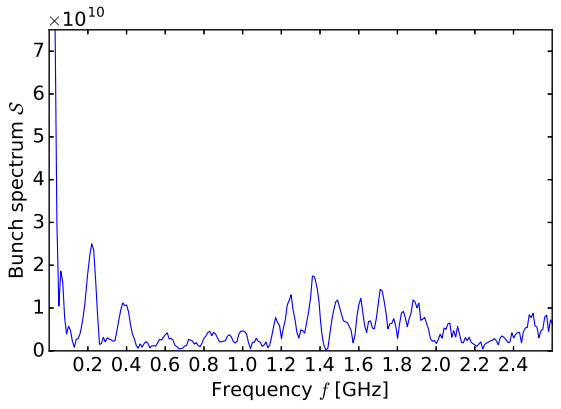

(f) Spectrum at $t=9.24 \mathrm{~ms}$

FIG. 3. Examples of a density modulation of a long bunch at injection in the SPS ( $p=26 \mathrm{GeV} / \mathrm{c})$ with the rf voltage switched off in the Q26 optics. The bunch profile (top), together with the corresponding bunch spectrum (bottom) at three different times after injection demonstrating the evolution of the instability.

impedance sources in the SPS are located at high frequencies $(f>100 \mathrm{MHz})$ in comparison with the bunch spectrum and lead to microwave-like instability.

We describe the fast microwave instability by solving the linearized Vlasov equation using the perturbation theory [9]. We consider a distribution $\psi$ in the longitudinal phase space $(\theta, \Delta E)$, where $\theta=\omega_{\text {rev }} \tau$ is the azimuthal coordinate of a particle in the ring, $\omega_{\text {rev }}=2 \pi f_{\text {rev }}$ is the revolution angular frequency, $\tau$ is the longitudinal coordinate of the particle in time and $\Delta E$ the relative energy of a given particle with respect to the beam energy $E$. The bunch profile for the bunch distribution $\psi$ is denoted $\lambda(\theta)$ and the corresponding bunch spectrum $\mathcal{S}(f)$. The bunch distribution is composed of an unperturbed part $\psi_{0}$ and a perturbation $\psi_{1}$, for which the spectra are respectively:

$$
\begin{gathered}
\mathcal{S}_{0}(n)=\frac{1}{2 \pi} \int_{-\pi}^{\pi}\left[\int_{-\infty}^{\infty} \psi_{0}(\theta, \Delta E) d(\Delta E)\right] e^{-j n \theta} d \theta, \\
\mathcal{S}_{1}(n)=\frac{1}{2 \pi} e^{-j \Omega t} \int_{-\pi}^{\pi}\left[\int_{-\infty}^{\infty} \psi_{1}(\theta, \Delta E) d(\Delta E)\right] e^{-j n \theta} d \theta,
\end{gathered}
$$

where $\Omega$ is the oscillation frequency of the perturbation, of which the imaginary part gives the growth rate of the instability, and the terms in square brackets are the unperturbed/perturbed bunch profiles $\lambda_{0,1}(\theta)$. Since the momentum spread is small, the debunching is slow and the unperturbed bunch spectrum can be considered stationary. The stationary bunch spectrum corresponds to the contribution at low frequencies $(f<100 \mathrm{MHz})$, while the perturbation corresponds to the time dependent modulations at high frequencies $(f>100 \mathrm{MHz})$.

With the rf voltage switched off, the particle motion is only affected by the induced voltage:

$$
V_{\text {ind }}(\theta)=-q N_{b} \omega_{\text {rev }} \sum_{n}\left[\mathcal{S}_{0}(n)+\mathcal{S}_{1}(n)\right] \mathcal{Z}(n) e^{j n \theta} .
$$

The bunch interacts with impedance sources, which in most of the cases can be described by a resonator model:

$$
\mathcal{Z}(f)=\frac{R}{1+j Q\left(\frac{f}{f_{r}}-\frac{f_{r}}{f}\right)},
$$

where $R$ is the shunt impedance and $Q$ the quality factor determining the decay time of the wake. For the high frequencies under consideration $f_{r} \tau_{L} \gg 1$, the induced voltage coming from the stationary bunch spectrum $\mathcal{S}_{0}$ in Eq. (6) is negligible. Therefore, the linearized Vlasov equation can be expressed as:

$$
\begin{aligned}
& \frac{\partial \psi_{1}}{\partial t}-\frac{\left(q \omega_{\text {rev }}\right)^{2}}{2 \pi} N_{b}\left[\sum_{n} \mathcal{S}_{1}(n) \mathcal{Z}(n) e^{j n \theta}\right] \frac{\partial \psi_{0}}{\partial(\Delta E)} \\
& +\frac{\eta \omega_{\text {rev }}}{\beta^{2} E} \Delta E \frac{\partial \psi_{1}}{\partial \theta}=0 .
\end{aligned}
$$

For bunches with small energy spread, during the linear stage of the instability, the injected bunch distribution can be treated as monoenergetic with $\psi_{0}(\theta, \Delta E)=\lambda(\theta) \delta(\Delta E)$, 


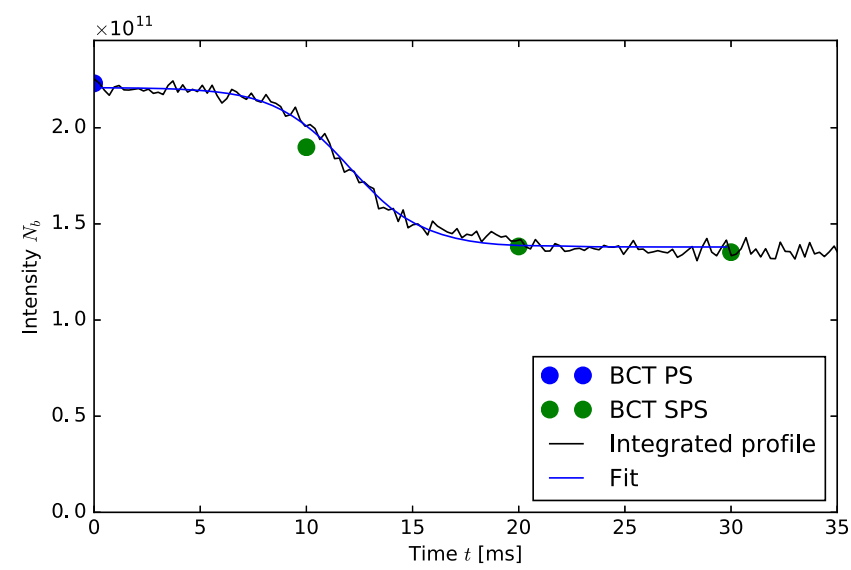

FIG. 4. The bunch intensity $N_{b}$ during the acquisition corresponding to Fig. 3 (Q26 optics). The bunch intensity was measured with a BCT at extraction from the PS (blue dot) and during acquisition in the SPS (green dots). The intensity is evaluated from the measured bunch profile and scaled to the measured intensity from PS (black), and fitted with a sigmoid function to be used in macroparticle simulations (blue).

where $\delta$ is the Dirac function. Solving the linearized Vlasov equation with this assumption leads to the matrix equation:
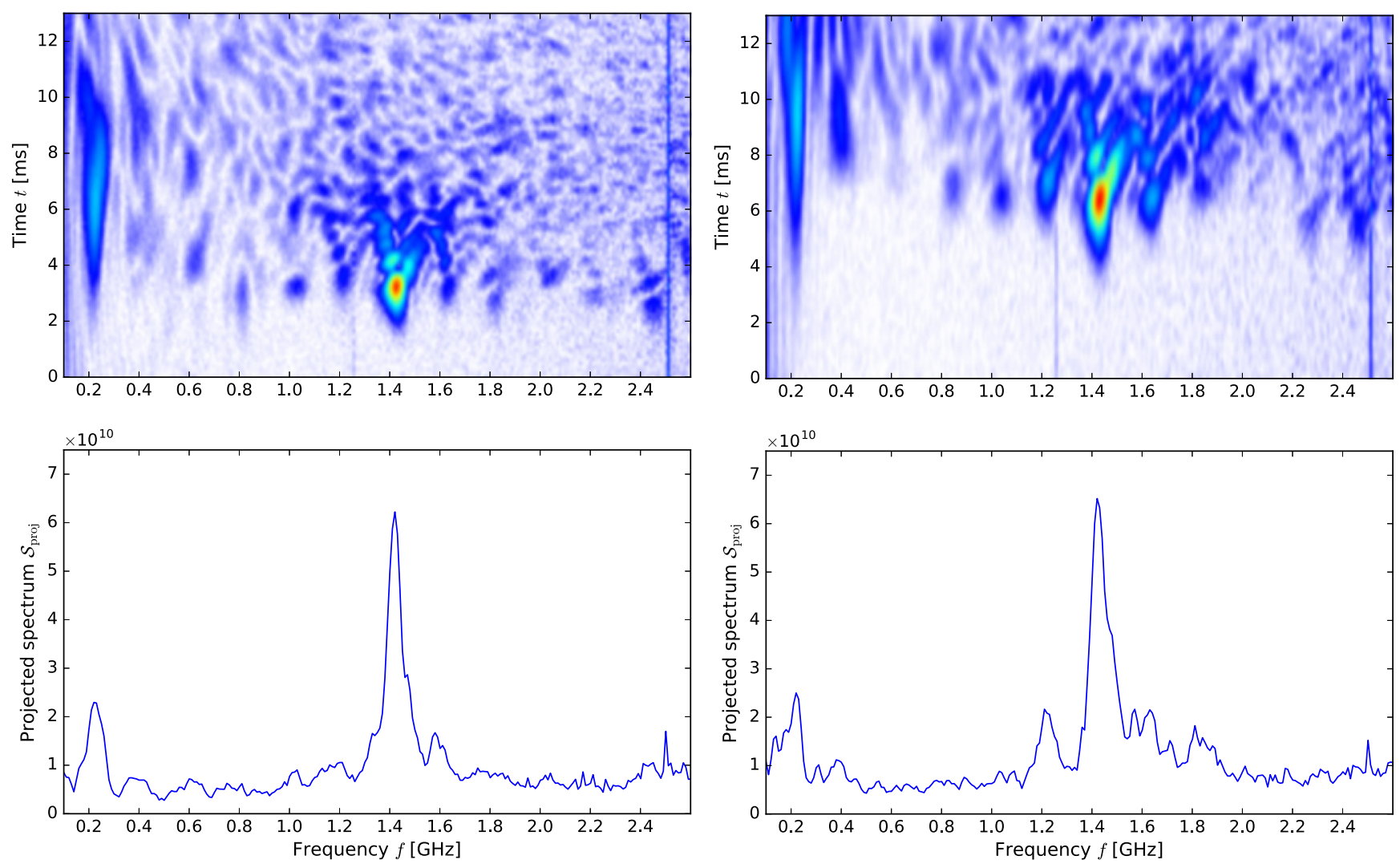

(a) Q20 optics
$\mathcal{S}_{1}(n)=-j \frac{\eta \omega_{\mathrm{rev}} N_{b}}{2 \pi \beta^{2} E}\left(\frac{q \omega_{\mathrm{rev}}}{\Omega}\right)^{2} n \sum_{n^{\prime}} \mathcal{S}_{0}\left(n-n^{\prime}\right) \mathcal{Z}\left(n^{\prime}\right) \mathcal{S}_{1}\left(n^{\prime}\right)$.

For narrow-band impedance sources (with the bandwidth $\left.\Delta \omega_{r}=\omega_{r} /(2 Q) \ll 1 / \tau_{L}\right)$ we can assume that the bunch spectrum is constant over the impedance width with $\mathcal{S}_{0}\left(n-n^{\prime}\right) \approx \mathcal{S}_{0}\left(n-n_{r}\right)$ where $n_{r}=f_{r} / f_{\text {rev }}$. In this case the spectrum of the unstable mode is

$$
\mathcal{S}_{1} \sim n \mathcal{S}_{0}\left(n-n_{r}\right) \sim n e^{-\frac{\sigma_{\text {rms }}^{2}\left(n-n_{r}\right)^{2}}{2}},
$$

where $1 / \sigma_{\mathrm{rms}}$ is the bandwidth of the stationary spectrum $\mathcal{S}_{0}$. This also defines the bandwidth of the unstable mode, which implies that a longer bunch gives a better resolution to the measured modulation. Note that the peak is shifted by $1 /\left(n_{r} \sigma_{\mathrm{rms}}^{2}\right)$ with respect to $n_{r}$, which will be shown in Sec. IV.

Finally, the growth rate of the instability is:

$$
\operatorname{Im} \Omega \approx 2 \pi f_{r}\left(\frac{q^{2} N_{b} \omega_{\mathrm{rev}} \eta}{16 \pi E} \frac{R}{Q}\right)^{\frac{1}{2}} .
$$

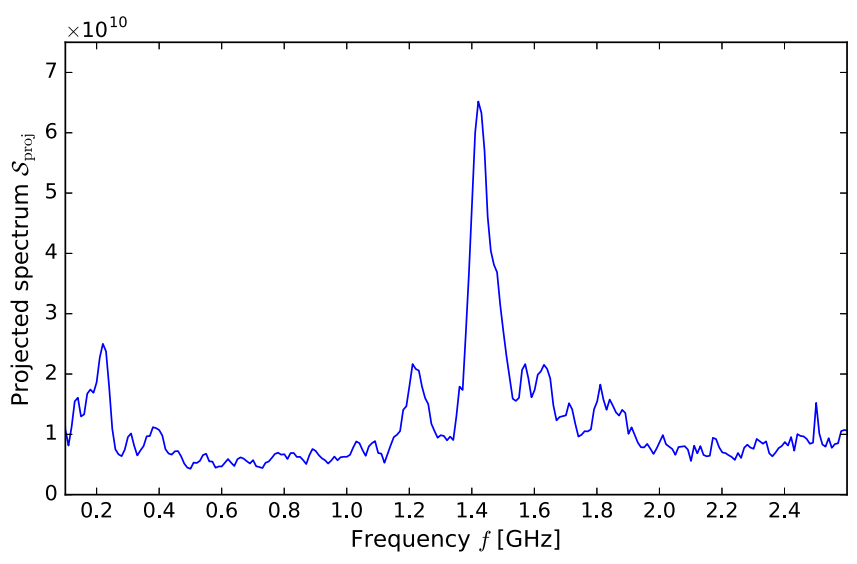

(b) Q26 optics

FIG. 5. Examples of the evolution of the bunch spectrum with time after injection (top) and the corresponding projected spectrum (bottom) in the Q20 (left) and Q26 (right) optics, for an injected bunch intensity $N_{b} \approx 2.1 \times 10^{11} \mathrm{ppb}$. The acquisition in the Q26 optics corresponds to the example shown in Fig. 3. 

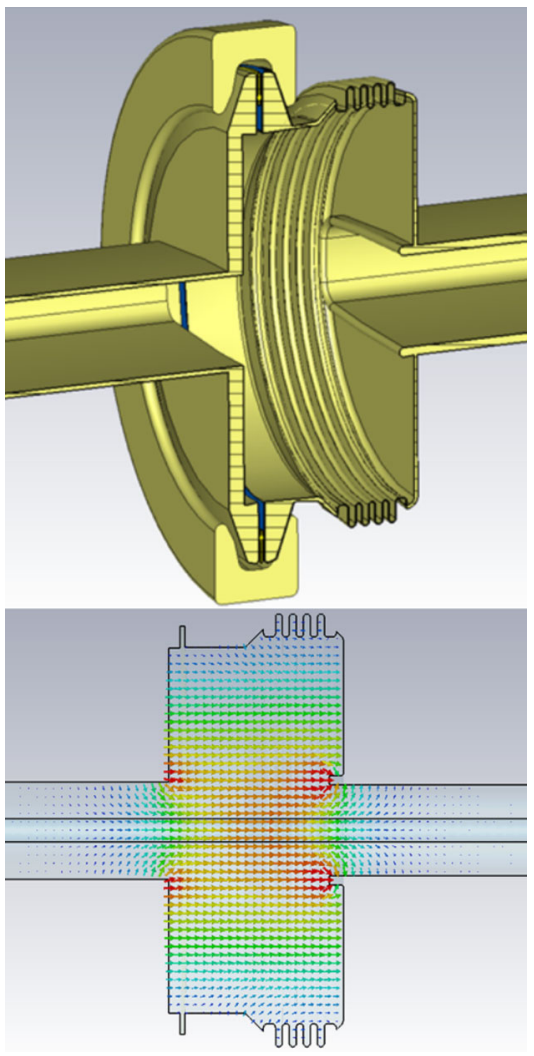

FIG. 6. The geometry of the QF-type vacuum flange and bellow as drawn using the CST [15] software (top), together with the field pattern of the main resonance at $1.4 \mathrm{GHz}$ corresponding to the pillbox cavity-like mode TM010.

The bunch profile modulation is mainly driven by impedance sources with high $R / Q$ and high resonant frequency $f_{r}$. Note also the dependence on the slippage factor $\eta$ that is relevant in the following discussion of results for the Q20 and Q26 optics.

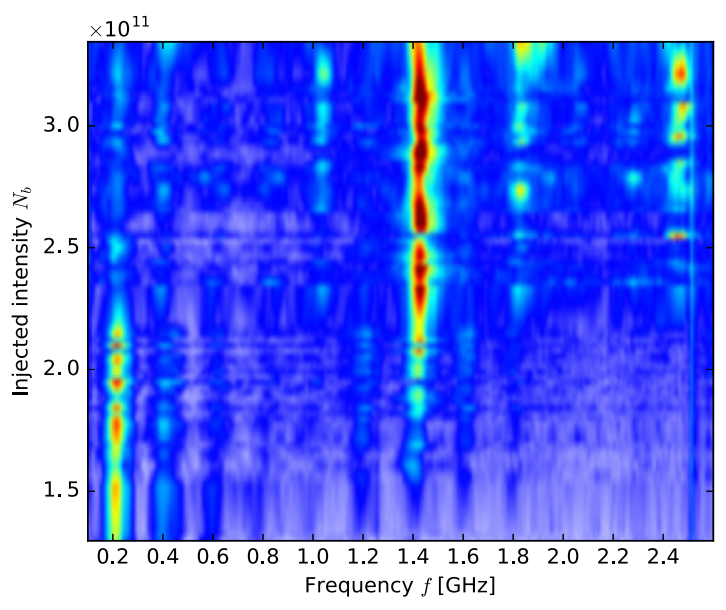

(a) Q20 optics

\section{MEASUREMENTS}

\section{A. Setup}

To measure the time dependence of the high frequency modulation, the bunch profile $\lambda(\tau)$ was measured at regular time interval using a wall current monitor (WCM) and the corresponding bunch spectrum $\mathcal{S}(f)$ was obtained using a fast Fourier transform (below the bunch spectrum is normalized to the bunch intensity). The perturbations of the measured bunch spectrum due to the various elements in the measurement line (e.g., cables) were corrected $[12,13]$. This correction is effective up to $\approx 2.0 \mathrm{GHz}$. Above this frequency, the amplitude of the corrected bunch spectrum may be overestimated. Below, we will consider the frequency range $0.1 \mathrm{GHz}<f<2.0 \mathrm{GHz}$.

Measurements were done for a broad range of bunch intensities $N_{b}$, which was measured using a DC beam current transformer (BCT) at the extraction time in the PS and during the acquisition in the SPS. Since the integration time of the BCT is long $(10 \mathrm{~ms})$ with respect to the measurement time scale $(30 \mathrm{~ms})$, the remaining information about the bunch intensity was obtained by integrating the measured bunch profiles, as shown in Fig. 4.

Two optics configurations are available in the SPS named Q20 and Q26 after the transverse tune. These are characterized by different transition Lorentz factors $\left(\gamma_{t, \mathrm{Q} 20} \approx 18\right.$ and $\left.\gamma_{t, \mathrm{Q} 26} \approx 22.8\right)$, resulting in a slippage factor $\eta$ that is 2.9 times higher for the Q20 optics than in the Q26 optics. Therefore, the debunching is also faster for the Q20 optics (see Eq. (3)) and measurements were done on a different time scale: over 600 turns in the machine for the Q20 optics and 1000 turns for the Q26 optics (the revolution period is $T_{\text {rev }} \approx 23.1 \mu \mathrm{s}$ ). On these time scales, the debunching is considered small.

According to Eq. (11), the growth rate of the bunch profile modulation is faster in the $\mathrm{Q} 20$ optics with respect to

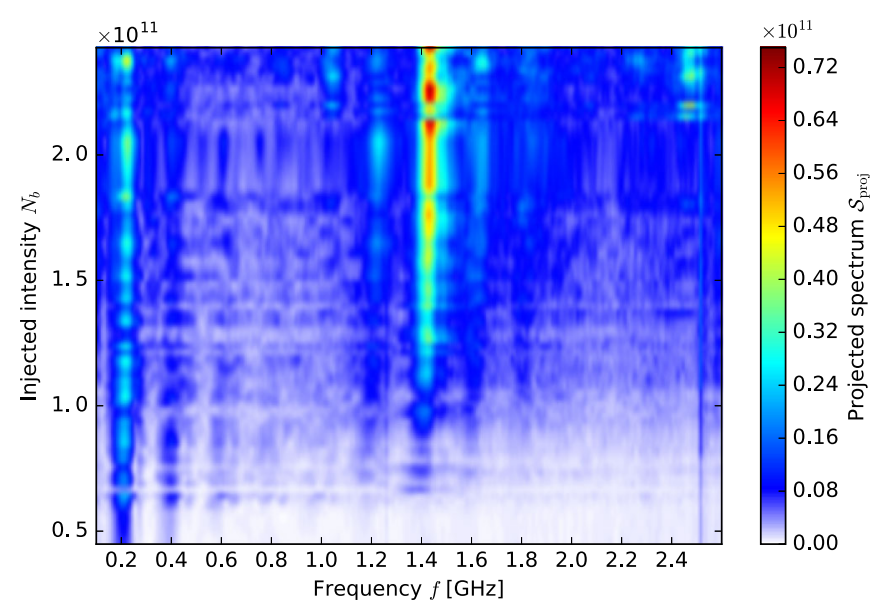

(b) Q26 optics

FIG. 7. Projected spectra as a function of intensity in Q20 (left) and Q26 (right) optics. The color scale corresponds to the amplitude of the projected spectrum. 
the Q26 optics. This was also observed in measurements where the bunch modulation is happening at a time scale $\sqrt{\eta_{\mathrm{Q} 20} / \eta_{\mathrm{Q} 26}} \approx 1.7$ faster in the $\mathrm{Q} 20$ optics, as shown in the upper plots of Fig. 5 (for same bunch intensity $N_{b} \approx 2.1 \times 10^{11} \mathrm{ppb}$ ). However, a higher slippage factor also implies a faster debunching which smears the modulation. Therefore, the intensity threshold was higher in the Q20 optics and measurements were done in a different intensity range, $N_{b} \approx(1.3-3.5) \times 10^{11} \mathrm{ppb}$ in the Q20 optics and $N_{b} \approx(0.4-2.5) \times 10^{11} \mathrm{ppb}$ in the Q26 optics.

Note that fast losses (within less than 1000 turns) were observed for high bunch intensities, as shown in Fig. 4, and at a lower intensity threshold in the Q26 optics. This may be the sign of possible transverse instabilities. In most cases, the modulations take place in a time scale smaller than the one of the intensity loss. Additionally, it is assumed for this study that there is no coupling of the transverse particle motion onto the longitudinal one.

\section{B. Data analysis}

For each acquisition, the projected spectrum $\mathcal{S}_{\text {proj }}(f)$ corresponding to the maximum value of the mode amplitude of $\mathcal{S}(f)$ along the time axis was used. Examples are shown in Fig. 5, where the evolution of the spectrum with time is represented in the upper plot and the corresponding projection is shown below. In these examples the dominant modulations (peaks) are those at $1.4 \mathrm{GHz}$ and $200 \mathrm{MHz}$. Smaller peaks at $1.2 \mathrm{GHz}$ and $1.6 \mathrm{GHz}$ are also visible.

In Fig. 7, the measured maximum spectra are presented as a function of the injected bunch intensity. All the vertical lines correspond to modulations that may be associated to a possible impedance source. The most straightforward to identify is the modulation at $200 \mathrm{MHz}$ corresponding to the main impedance of the TWC rf system, which has the highest resistive impedance in the machine. However, the most significant modulation is the one at $1.4 \mathrm{GHz}$ both in the Q20 and Q26 optics. When these measurements were done, the responsible impedance source was still unknown. It was identified later, after a thorough survey of the SPS elements [14], this was due to the QF-type vacuum flanges shown in Fig. 6. The vacuum flanges are indeed the biggest impedance source in terms of $R / Q$ at high frequencies in the present SPS impedance model and are expected to be the main source of microwave instability.

Another peak, present in both optics, is located at $2.45 \mathrm{GHz}$ and can be associated with the impedance of the QD-type vacuum flanges. They do not have the highest impedance in terms of $R / Q$, but still can generate a significant modulation due to their high resonant frequency $f_{r}$, according to Eq. (11). However, as discussed in the Sec. III A, the correction of the measurement line transfer function may give an overestimation of the spectrum above $2 \mathrm{GHz}$. Therefore, although this peak is present its absolute amplitude may not be accurate.

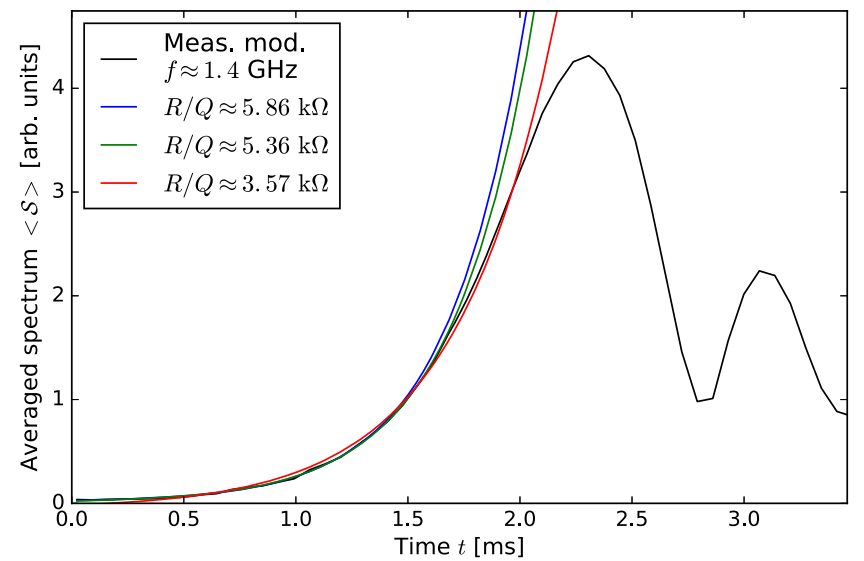

FIG. 8. The measured spectrum amplitude $\langle\mathcal{S}\rangle$ averaged over a bandwidth of $\pm 50 \mathrm{MHz}$ around $1.4 \mathrm{GHz}$ during the initial phase of the instability in the Q20 optics for $N_{b} \approx 2.7 \times 10^{11} \mathrm{ppb}$ (black). The growth of the instability on the early stage was fitted using an exponential function for a time: $t_{\text {fit }}=1.4 \mathrm{~ms}$ (blue), $t_{\text {fit }}=1.6 \mathrm{~ms}$ (green) and $t_{\text {fit }}=2.0 \mathrm{~ms}$ (red). The corresponding value of $R / Q$ is computed using Eq. (11).

Close to the peak at $1.4 \mathrm{GHz}$, other lines are present at multiples of $200 \mathrm{MHz}$ : at $1.2 \mathrm{GHz}$ and $1.6 \mathrm{GHz}$ in the bunch intensity range $(1.5-2.0) \times 10^{11} \mathrm{ppb}$, and at $1.0 \mathrm{GHz}$ and $1.8 \mathrm{GHz}$ for bunch intensities above $2.3 \times 10^{11} \mathrm{ppb}$. All these peaks can be associated with various resonant frequencies of the QF-type and QD-type vacuum flanges impedance, except the one at $1.0 \mathrm{GHz}$ where no major contribution was identified. However, these peaks are correlated to the main ones at $200 \mathrm{MHz}, 1.4 \mathrm{GHz}$ and $2.45 \mathrm{GHz}$. For instance, the lines at $1.2 \mathrm{GHz}$ and $1.6 \mathrm{GHz}$ are correlated with both peaks at $200 \mathrm{MHz}$ and $1.4 \mathrm{GHz}$. Concerning the lines at $1.0 \mathrm{GHz}$ and $1.8 \mathrm{GHz}$, they are correlated with the peaks at $1.4 \mathrm{GHz}$ and $2.45 \mathrm{GHz}$. Therefore, these smaller peaks can be nonlinear products of the main modulations and may not be caused

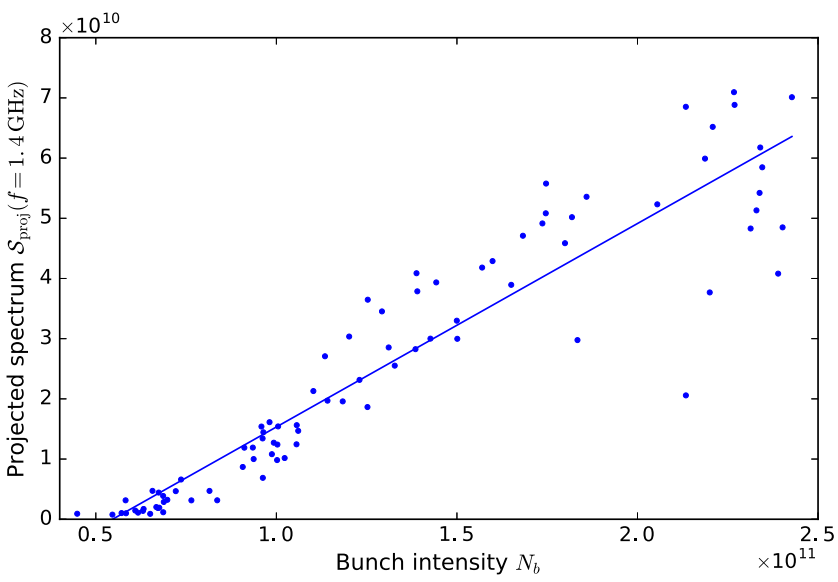

FIG. 9. Maximum spectrum at $1.4 \mathrm{GHz}$ as a function of the bunch intensity $N_{b}$ in the Q26 optics. Measurements are fitted linearly and $b_{\text {peak }} \approx 0.34$ is the slope of the fit. 


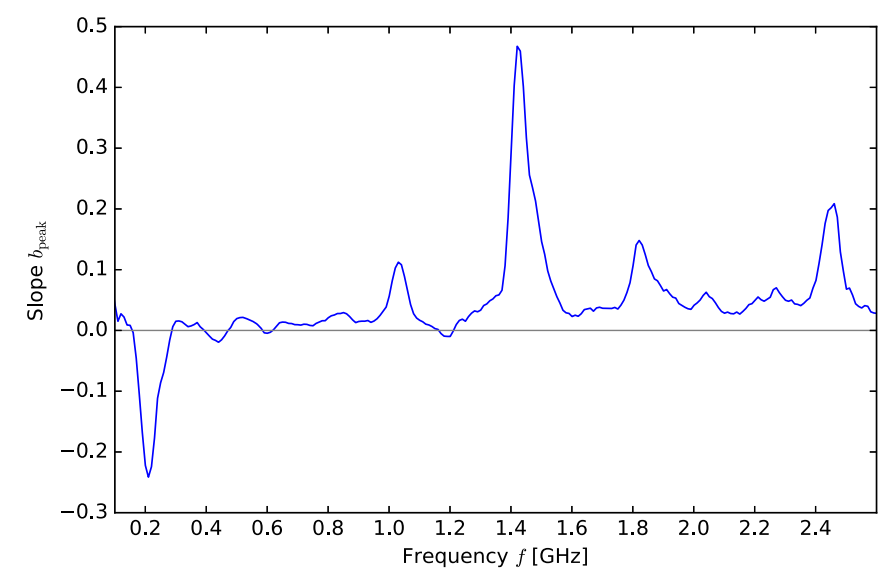

(a) Q20 optics

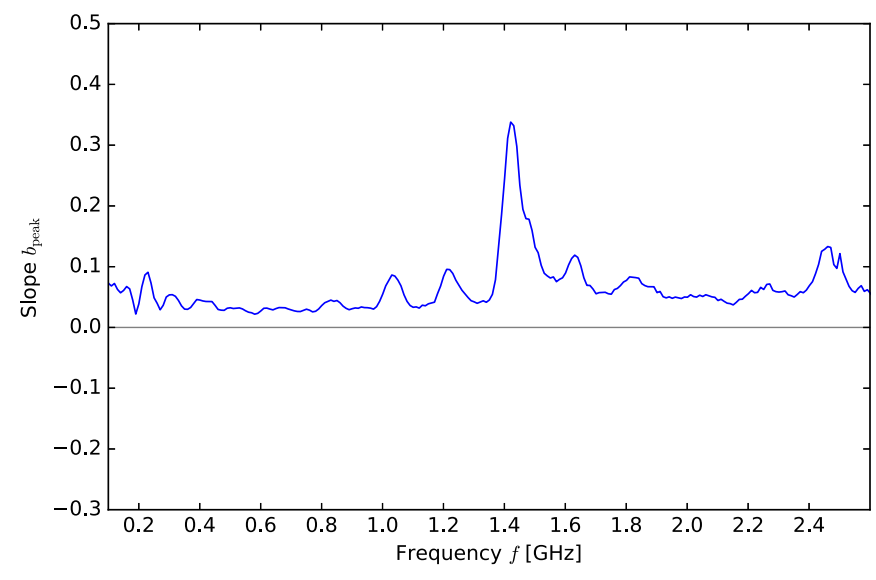

(b) Q26 optics

FIG. 10. The slope $b_{\text {peak }}$ as a function of frequency for the Q20 (left) and Q26 (right) optics. The value of the slope at $1.4 \mathrm{GHz}$ in the plot (b) correspond to the example shown in Fig. 9 with $b_{\text {peak }} \approx 0.34$.

by any impedance source. Another feature is the absence of peak corresponding to the TWC at $800 \mathrm{MHz}$. Despite its high $R / Q$, it appears to be located at the resonant frequency $f_{r}$ that is too small to drive a modulation in competition with other impedances. This implies that some significant impedance sources may not be identified with this method.

It is possible to estimate the values of $R / Q$ using Eq. (11) from the instability growth rate measured well above the instability threshold. An example is shown in Fig. 8 for an acquisition made in the Q20 optics for a bunch intensity $N_{b} \approx 2.7 \times 10^{11} \mathrm{ppb}$ and for the signal growing at $1.4 \mathrm{GHz}$. The initial phase of the instability can be described by the linear theory with the exponential signal growth. With the increase of the momentum spread the instability saturates due to nonlinearities. The calculated growth rate depends on the time scale taken for the fit and results may change with a variation of approximately $\pm 20 \%$ (see Fig. 8) due to the quadratic dependence of the calculated $R / Q$ on $\operatorname{Im} \Omega$. In addition, the shot-to-shot variation (from one acquisition to another) leads to significant error-bars. The calculated values of the impedance from the growth rate are $R / Q \approx(5 \pm 3) \mathrm{k} \Omega$ for the Q20 optics and $R / Q \approx(7 \pm 3) \mathrm{k} \Omega$ for the Q26 data. On average these values are in good agreement with the ones obtained from electromagnetic simulations and measurements for the QF-type vacuum flanges which have two main resonances: $R / Q \approx 6 \mathrm{k} \Omega$ at $1.415 \mathrm{GHz}$ and $R / Q \approx 1.8 \mathrm{k} \Omega$ at $1.395 \mathrm{GHz}[8]$.

The measured maximum amplitude of the projected spectrum $\mathcal{S}_{\text {proj }}$ is a more convenient parameter for comparison with results of macroparticle simulations. The results shown in Fig. 7 are simplified by fitting the peaks as a function of the bunch intensity $N_{b}$ (comparisons of $2 \mathrm{D}$ plots are easier than 3D plots). An example is shown in Fig. 9, where the peak at $1.4 \mathrm{GHz}$ for the results in the Q26 optics are fitted linearly as a function of intensity. The slope of the linear fit is noted $b_{\text {peak }}$ (shortened to slope $b_{\text {peak }}$ below). This is done for each frequency, and the results are shown in Fig. 10. All the peaks discussed above at multiples of $200 \mathrm{MHz}$ are visible in Fig. 10. Note that the slope $b_{\text {peak }}$ at $200 \mathrm{MHz}$ is negative in the Q20 optics, due to the fact that this peak is present only for small bunch intensities [see Fig. 7(a)]. This definition will be used for comparison with macroparticle simulations in Sec. IV.

A byproduct that can be extracted from these measurements is the bunch energy loss in the absence of rf voltage. The bunch energy variation per turn due to the resistive machine impedance is given by [16]:

$$
\delta E_{b}=-\frac{\left(q N_{b}\right)^{2} \omega_{\text {rev }}}{\pi} \sum_{n=0}^{\infty} \operatorname{Re} \mathcal{Z}(n)|\mathcal{S}(n)|^{2} .
$$

In the present configuration, the loss rate also depends on the unstable bunch spectrum, which is sampling the high

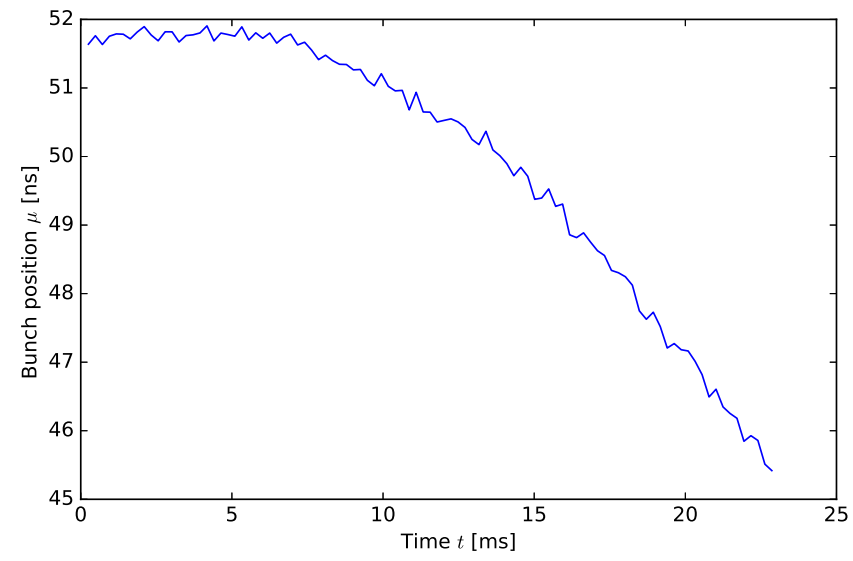

FIG. 11. Bunch position $\mu$ drift with time during the acquisition due to the energy loss corresponding to the acquisition in Fig. 3 in the Q26 optics and a bunch intensity $N_{b} \approx 2.1 \times 10^{11} \mathrm{ppb}$. 


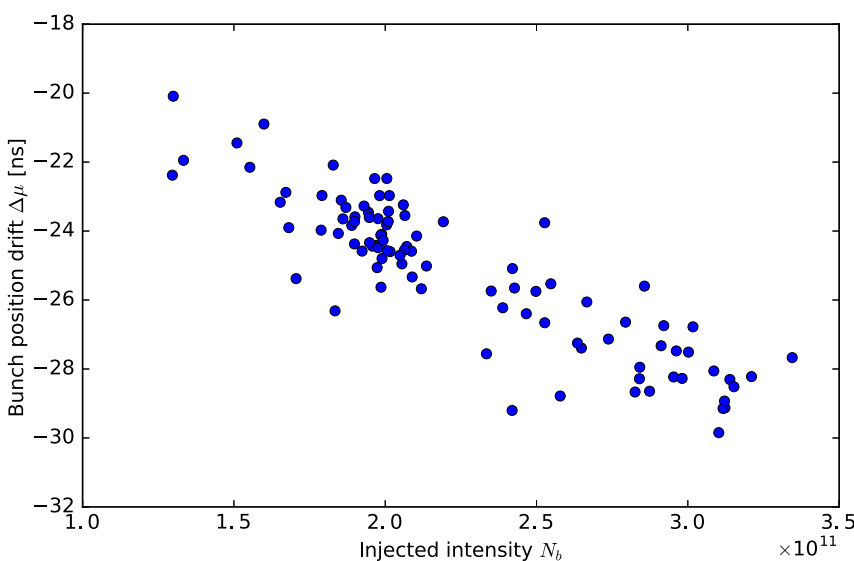

(a) Q20 optics

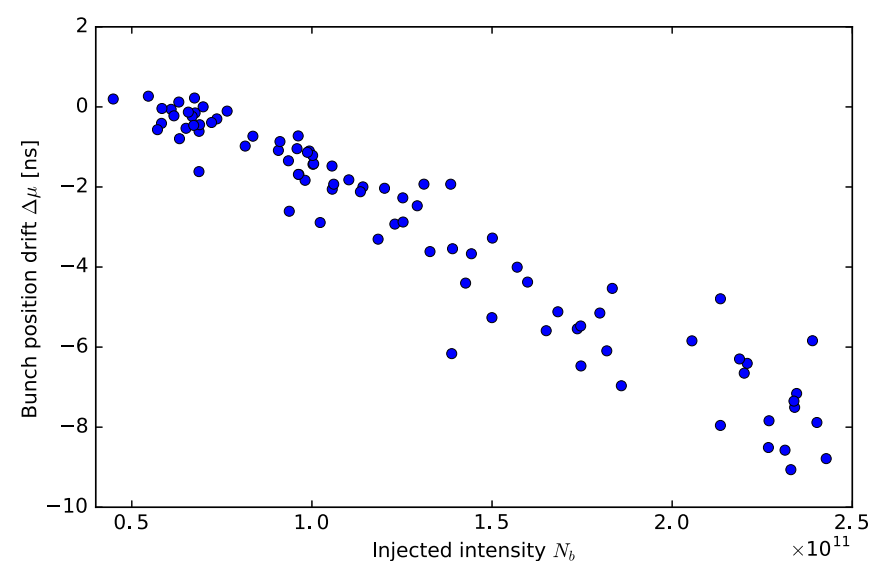

(b) Q26 optics

FIG. 12. Measured drift in bunch position $\Delta \mu$ as a function of the injected bunch intensity $N_{b}$ in the Q20 (left) and Q26 (right) optics, respectively after 600 and 1000 turns.

frequency impedance sources driving the instability. The bunch profile is measured by the wall current monitor with a regular time interval corresponding to a fixed number of turns in the ring. The position of the bunch center of mass $\mu$ should stay constant assuming that the bunch does not lose energy, and that the acquisition frequency is synchronous with the revolution frequency $f_{\text {rev }}$. With the rf voltage switched off, the energy loss manifests through a drift in time of the bunch position $\mu$. Since measurements are done above transition energy in the SPS, the revolution frequency of the bunch gradually increases while the bunch loses energy. Therefore the bunch position $\mu$ decreases with time as shown in Fig. 11.

To analyze the bunch energy loss as a function of the bunch intensity, we take the drift in bunch position $\Delta \mu$ during the time acquisition time $\Delta t$ (600 turns in the Q20 optics and 1000 turns in the Q26 optics). The measured drift in bunch position as a function of the bunch intensity is shown in Fig. 12. For zero bunch intensity, the drift in bunch position should be zero since the bunch would not lose energy and its revolution period would remain constant. Due to the momentum spread, we would only see a slow debunching. For the measurements in the Q20 optics in Fig. 12(a), there is an offset in $\Delta \mu$ for small intensities, which can be explained by a small mismatch in the initial bunch energy with respect to the expected one at injection. In Sec. IV, these results will be compared with macroparticle simulations to test the energy loss due to the effective resistive impedance of the machine.

\section{PARTICLE SIMULATIONS}

\section{A. BLonD simulations}

To be able to extract more accurate information from the measurements presented above, macroparticle simulations were done taking into account the non-linear effects and a realistic bunch distribution in energy. The simulation code BLoND [17] was used including the present impedance model shown in Fig. 2. The SPS machine parameters were set in the simulations to match the ones in measurements. To cover the same range in bunch intensity and injected bunch length, each acquisition was reproduced in simulations. The initial macroparticle distribution was generated along the longitudinal and the momentum coordinates independently. This was done in order to generate exactly the same bunch profiles as in measurements since the instability growth depends on the overlap of the bunch spectrum and the high frequency impedance. As discussed in Sec, III A, the correction of the measurement line transfer function includes some unphysical noise above $2 \mathrm{GHz}$. Therefore, the input bunch profile was smoothed using a Chebyshev filter (type II) with the cutoff frequency set at $2.0 \mathrm{GHz}$.

The particle distribution in momentum was generated using a parabolic function with the expected rms momentum spread $\Delta p_{m} / p$ (corresponding to the bunch length $\tau_{L}$ ) and assuming that in the PS before extraction the bunch was matched to the rf bucket with intensity effects. To take into account the effect of the potential-well distortion in the PS, a constant reactive impedance with $\operatorname{Im} \mathcal{Z} / n=18.4 \Omega$ [18] was assumed. In each simulation, the bunch intensity was set taking into account the realistic losses estimated by using a fit to the measured intensity as shown in Fig. 4. Convergence tests were performed to ensure that the results are not affected by numerical noise. The number of macroparticles was scanned from 2 million to 50 million and convergence was reached well below 40 million which were used for simulations presented here. Intensity effects are computed once per turn in the synchrotron with a resolution in time domain for the wakefields adjusted to $78 \mathrm{ps}$. Therefore the impedance is sampled up to $6.4 \mathrm{GHz}$, covering the frequency range of interest $(0-2.5 \mathrm{GHz})$. The tests the simulations were also performed for finer resolution in time, without changing the results if the number of particles is scaled accordingly. 


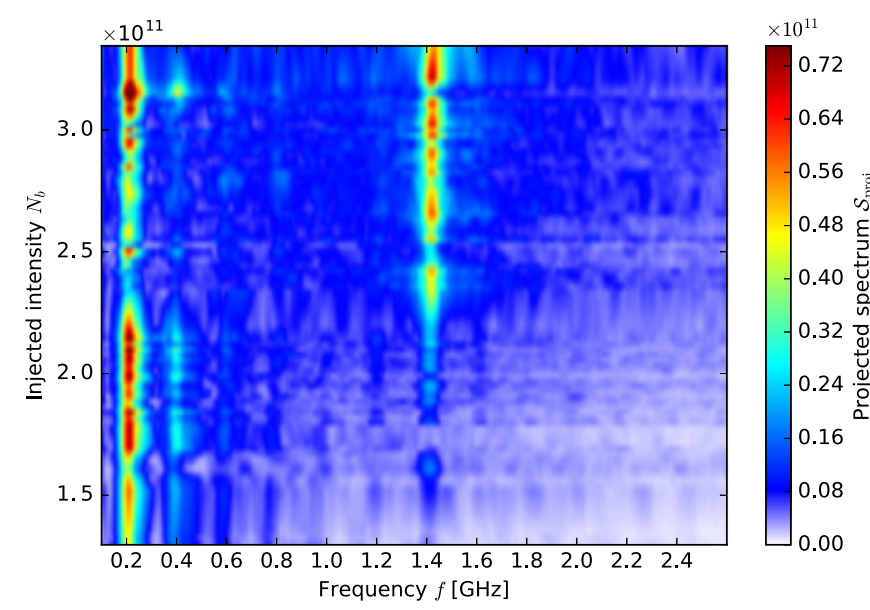

(a) Q20 optics

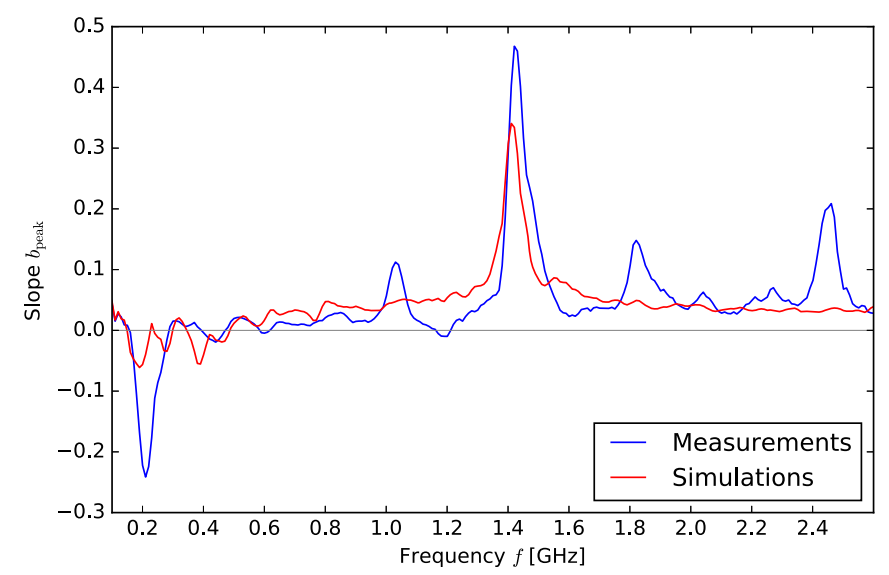

(c) Q20 optics

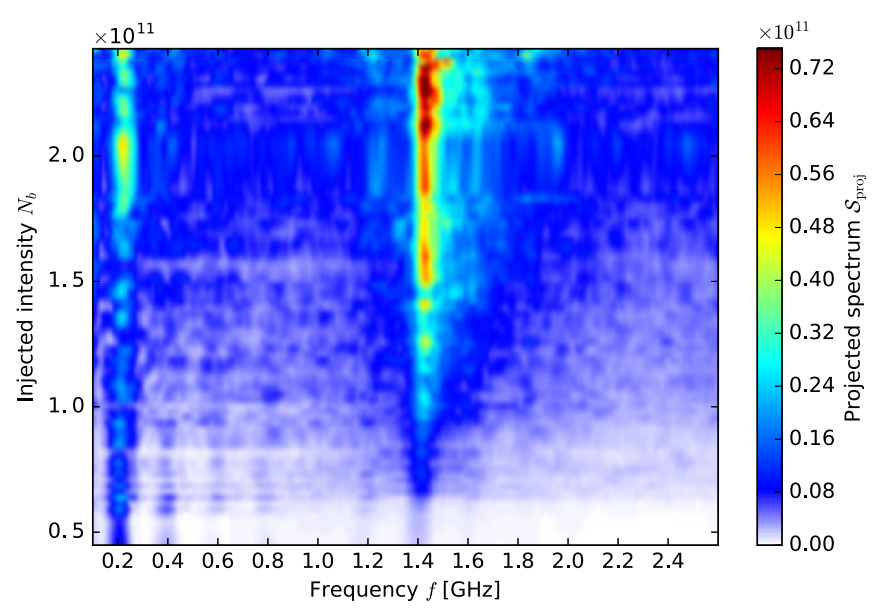

(b) Q26 optics

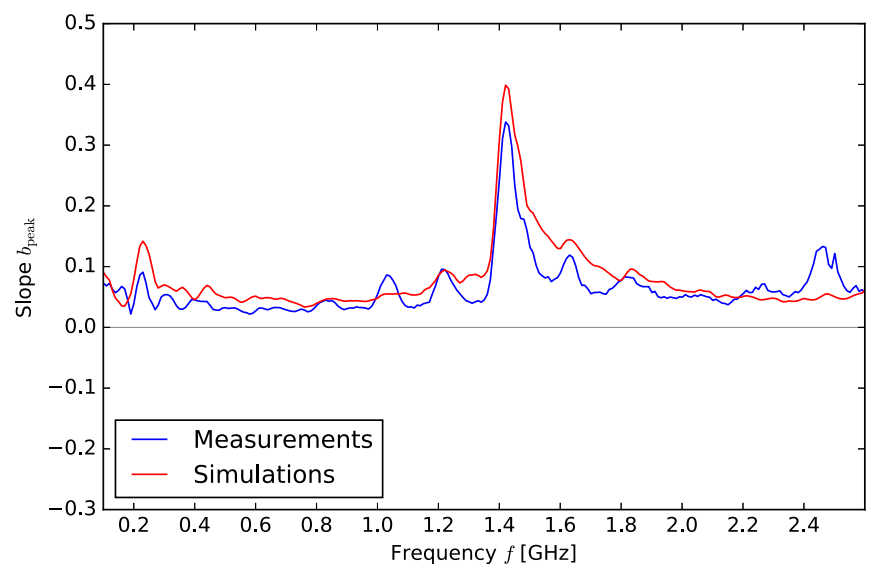

(d) Q26 optics

FIG. 13. Projected spectra $\mathcal{S}_{\text {proj }}$ from simulations as a function of intensity (top), and the corresponding slope $b_{\text {peak }}$ as a function of frequency (bottom) for both Q20 (left) and Q26 (right) optics. The simulated slopes $b_{\text {peak }}$ in red are compared with the measured ones in blue.

The analysis of simulation results was done using the same method as for measurements and results are shown in Fig. 13. The peak at $1.4 \mathrm{GHz}$ due to the impedance of the QF-type vacuum flanges is reproduced both in the Q20 and Q26 optics. The intensity threshold of this instability is in excellent agreement with the measurements $\left(N_{\text {th,Q20 }} \approx 2.2 \times 10^{11} \mathrm{ppb}\right.$ and $\left.N_{\text {th,Q26 }} \approx 1.0 \times 10^{11} \mathrm{ppb}\right)$, as well as the slopes $b_{\text {peak }}$ for the Q26 optics. We also note the absence of modulation at $800 \mathrm{MHz}$ from the TWC rf system, like in measurements. An interesting observation is that the position of the peaks are not exactly centered in $200 \mathrm{MHz}$ and $1.4 \mathrm{GHz}$, but slightly off-setted at higher frequencies (about $20 \mathrm{MHz}$ ). However, the input parameters of the impedance sources of the main rf system and the QF-type vacuum flanges are well centered at $200 \mathrm{MHz}$ and 1.4 MHz. This was expected from Eq. (10), where it was shown that the center of the unstable spectrum should be shifted by $1 /\left(n_{r} \sigma_{\mathrm{rms}}^{2}\right)$. This shift is also present in measurements (see Fig. 8).
We can notice deviations between measurements and simulations in the slope $b_{\text {peak }}$. The slope $b_{\text {peak }}$ at $1.4 \mathrm{GHz}$ for the Q20 optics is lower in amplitude than in measurements. Moreover, the various peaks are in competition with each other. A side-effect of the too low peak at $1.4 \mathrm{GHz}$ is that the peak at $200 \mathrm{MHz}$ is not reduced at high intensities in simulations. Concerning the peaks at multiples of $200 \mathrm{MHz}$ around $1.4 \mathrm{GHz}$, they are barely visible in the Q26 optics simulations since they are below the noise background and are absent in simulations for the Q20 optics.

The drift in bunch position due to the energy loss discussed above was also calculated in simulations and results are shown in Fig. 14. For the Q26 optics, the drift in bunch position in simulations is in excellent agreement with the measurements. For the Q20 optics, an energy mismatch of $-20 \mathrm{MeV}$ is required in simulations to get the same offset as in the measured drift in bunch position $\Delta \mu$ for low intensities. Above the intensity threshold for the 


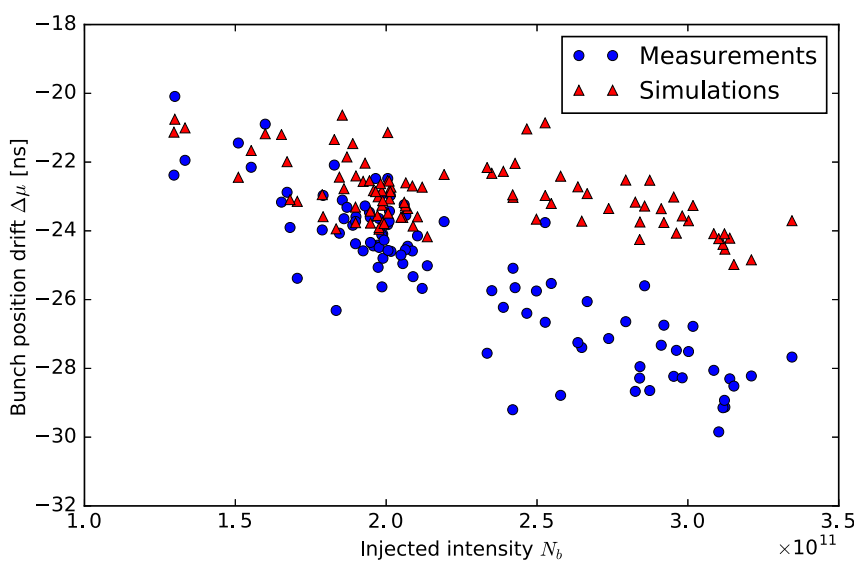

(a) Q20 optics

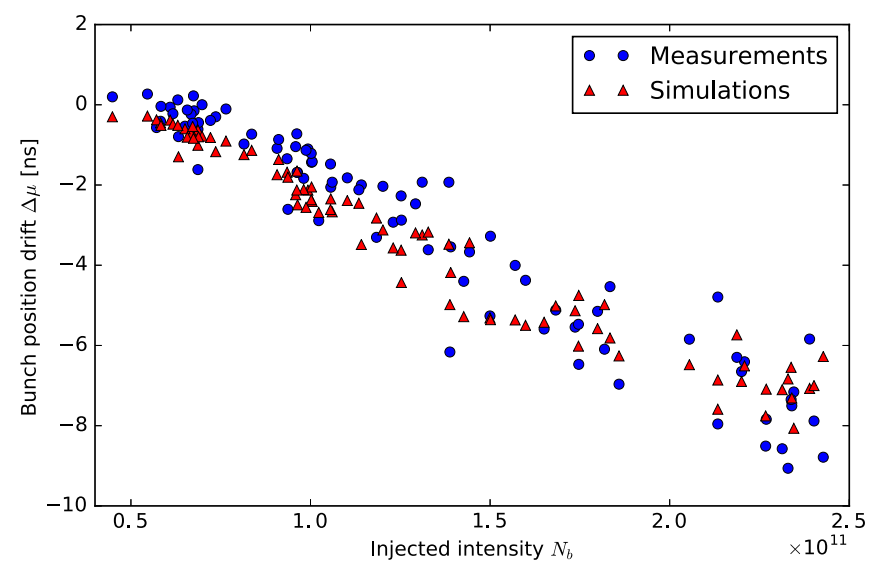

(b) Q26 optics

FIG. 14. The simulated (red) drift in bunch position $\Delta \mu$ compared with the measured one (blue) in the Q20 (left) and the Q26 (right) optics.

peak at $1.4 \mathrm{GHz}\left(N_{\mathrm{th}, \mathrm{Q} 20} \approx 2.2 \times 10^{11} \mathrm{ppb}\right)$, the drift in bunch position deviates from the measured one.

The main goal of these measurements was to identify impedance sources that could drive microwave instabilities, which has been achieved. Indeed, the measured modulation at $1.4 \mathrm{GHz}$ was identified to be driven by the impedance of the QF-type vacuum flanges and the effect was reproduced in simulations, implying that the evaluation of the impedance in terms of $R / Q$ is reasonable. This is further supported by the good agreement in the measured and simulated drifts in bunch position in the Q26 optics. Concerning the Q20 optics, the main peaks are also reproduced but with some non-negligible deviations. Since the time scale of the development of the instability and of debunching is very short, small differences in the initial particle distribution between measurements and simulations could lead to a significant discrepancy in results. Therefore, the initial bunch distribution was varied to investigate the origin of the deviations.

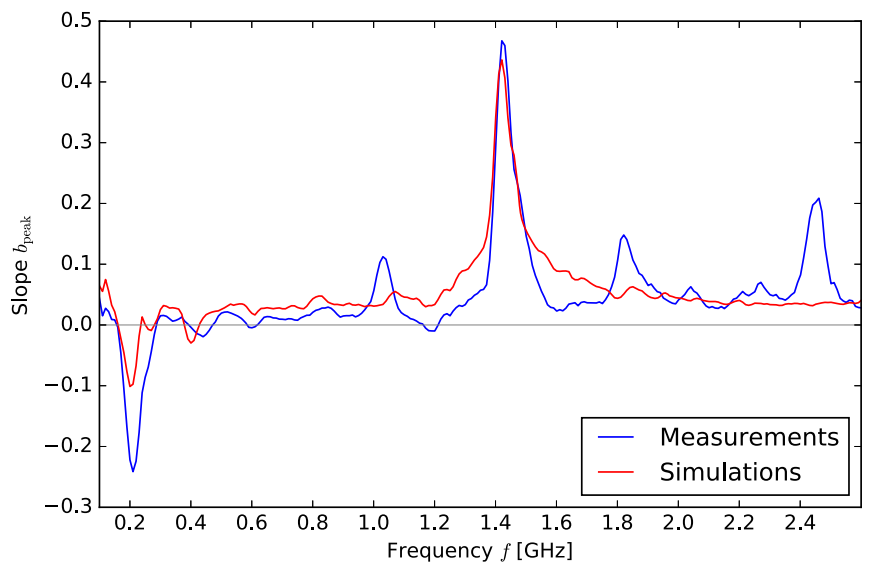

(a) Q20 optics

\section{B. Effect of the initial bunch distribution}

In simulations, to generate the distribution in momentum, we assumed that the bunch is matched to a PS non accelerating rf bucket modified by the induced voltage from a constant reactive impedance $\operatorname{Im} \mathcal{Z} / n$. However, some beam manipulations done in the PS just before extraction may change the shape of the rf bucket (e.g., extraction bump). This implies that the momentum spread may be lower than the previous estimation. Additionally, the resistive part of the PS impedance was neglected, although it should also reduce the previous estimation of the momentum spread for a given bunch length. With these assumptions, the momentum spread was reduced by $\approx 10 \%$ to evaluate what would be the effect on the instability, and results are shown in Fig. 15. A better agreement with measurements is reached in the Q20 optics for the peak at $1.4 \mathrm{GHz}$ with this condition, without affecting the results in the Q26 optics.

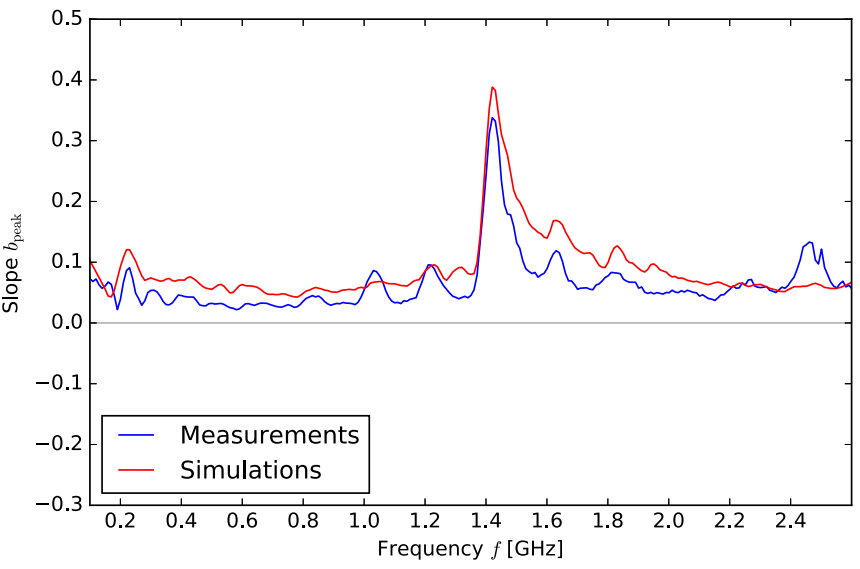

(b) Q26 optics

FIG. 15. The simulated slope $b_{\text {peak }}$ (red) compared to the measured one (blue) in the Q20 (left) and the Q26 (right) optics, with a reduction of the initial bunch momentum spread $\Delta p_{m} / p$ by $\approx 10 \%$ with respect to the spread used in simulations shown in Fig. 10 . 


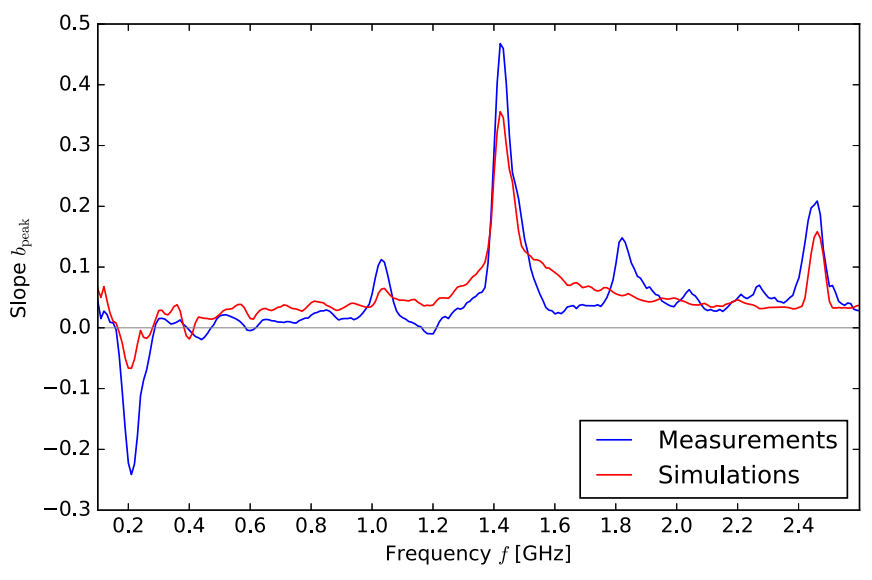

(a) Q20 optics

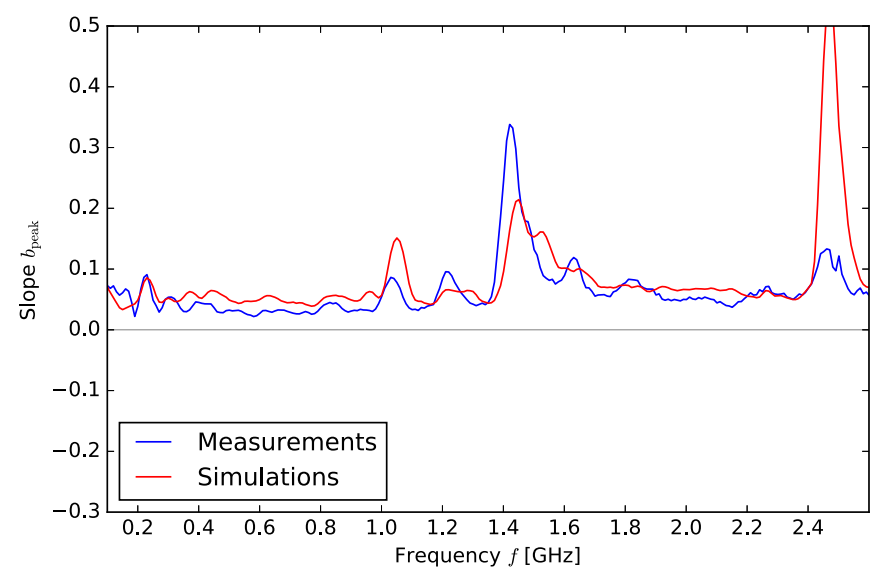

(b) Q26 optics

FIG. 16. The simulated slopes $b_{\text {peak }}$ (red) using an initial bunch profile with nonfiltered noise above $2 \mathrm{GHz}$ and a reduced momentum spread $\Delta p_{m} / p$ with respect to the simulations shown in Fig. 13, compared to the measured one (blue) in the Q20 (left) and Q26 (right) optics.

For the simulations presented above, the bunch profile was smoothed above $2 \mathrm{GHz}$ to remove the unphysical noise added from the correction of the measurement line transfer function (see Sec. IVA). Therefore, the effect of the impedance sources above $f>2 \mathrm{GHz}$ was significantly reduced in simulations and could not be reproduced the peak in measurements at $2.45 \mathrm{GHz}$. To evaluate the impact of high frequency impedance sources, simulations were done keeping the high frequency noise and with the reduction of the momentum spread. Results are shown in Fig. 16. A peak driven in simulations by the impedance of the QD-type vacuum flanges at $2.45 \mathrm{GHz}$ like in measurements. However, the amplitude of the slope $b_{\text {peak }}$ at $2.45 \mathrm{GHz}$ is much higher in simulations than in measurements, implying that the initial high frequency noise in the bunch spectrum is indeed overestimated.
Nevertheless, we can still associate the peak at $2.45 \mathrm{GHz}$ to the impedance of the QD-type vacuum flanges. Moreover, an interesting result is the peak at $1.0 \mathrm{GHz}$ appearing in simulations only with the presence of the modulation at $2.45 \mathrm{GHz}$ for the Q26 optics. The same result was obtained in the Q20 optics simulations by further reducing the momentum spread $\Delta p_{m} / p$ of the initial distribution by $10 \%$. There is no major impedance source at $1 \mathrm{GHz}$ in the SPS impedance model used in macroparticle simulations. Therefore this peak in the bunch spectrum is the non-linear product of the modulation of the bunch profile at several different frequencies. This implies that not all peaks measured with this method are driven by impedance sources, and this may be applicable to the other peaks at multiples of $200 \mathrm{MHz}$ around $1.4 \mathrm{GHz}$. Finally, the peak at $1.8 \mathrm{GHz}$ in the Q20 optics (see

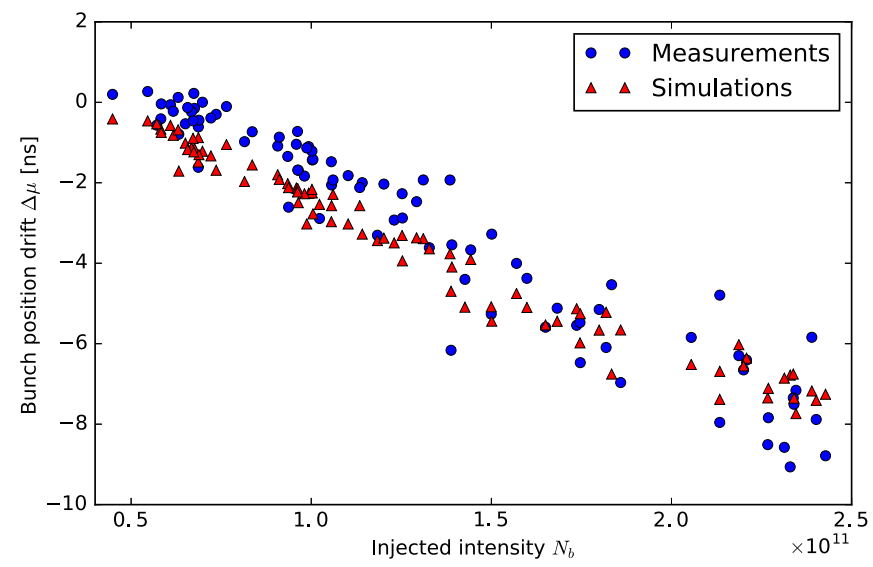

(b) Q26 optics

FIG. 17. The simulated drift in bunch position $\Delta \mu$ (red) using an initial bunch profile with nonfiltered noise above 2 GHz and a reduced momentum spread $\Delta p_{m} / p$ with respect to the simulations shown in Fig. 14, compared to the measured one (blue) in the Q20 (left) and Q26 (right) optics. 
Fig. 16(a)) was also never reproduced in simulations regardless of the initial bunch distribution. This may indicate that some additional impedance source may still not be identified or the present one (from the QD-type vacuum flanges) is underestimated in the present SPS impedance model. The impedance $R / Q$ of the impedance at $1.8 \mathrm{GHz}$ is nonetheless expected to be a small contribution in comparison to the QF-type vacuum flanges main resonance at $1.4 \mathrm{GHz}$.

The drift in bunch position $\Delta \mu$ was calculated again from simulations done with a smaller momentum spread and the unfiltered noise in the bunch profile above $2 \mathrm{GHz}$, and results are shown in Fig 17. A better agreement with measurements is reached for the Q20 optics, due to the larger energy loss caused by the stronger overlap between the unstable bunch spectrum and the resistive impedance during the instability. Some deviations between measurements and simulations are still present above $2.3 \times 10^{11} \mathrm{ppb}$ and indicate that the modulation at $1.8 \mathrm{GHz}$ missing in simulations and driven by an impedance source yet to be identified may contribute to the energy loss. A further reduction of the momentum spread by $10 \%$ also improves the simulated drift in bunch position with respect to measurements. It implies that a better knowledge of the initial bunch distribution is necessary to draw conclusions on potential impedance missing in the model.

\section{CONCLUSIONS}

The measurements of the bunch profile modulation by high frequency impedance sources with the rf voltage switched off allowed the identification of the main contributions driving microwave instabilities in the SPS. Measurements were performed in two optics available in the SPS, and a large bunch profile modulation was measured at $1.4 \mathrm{GHz}$. The QF-type vacuum flanges were then identified as the source of this instability. They are also expected to be the main source of microwave instabilities in the SPS with rf on [5]. Peaks in the spectrum at other frequencies were also studied, and allowed the identification of the QD-type vacuum flanges as the source of a bunch modulation at $2.45 \mathrm{GHz}$. Measurements were compared with macroparticle simulations to evaluate the accuracy of the present impedance model, and the reasonable agreement between measurements and simulations led to the conclusion that the main contributions of the vacuum flanges impedance are well represented. This method is very sensitive to the initial bunch distribution and a better agreement between measurements and simulations can be achieved with reduced momentum spread and depending on the initial high frequency noise in the bunch spectrum. The most important contributions to microwave instability have been identified and an impedance reduction of the impedance sources is foreseen in the frame of the LIU project.

\section{ACKNOWLEDGMENTS}

We would like to thank H. Damerau for setting up the rf parameters in the PS to provide long bunches. We would like to thank the impedance working group at CERN for the development of the SPS impedance model and in particular J. Varela, T. Kaltenbacher, C. Zannini, B. Salvant, and A. Farricker. We are grateful to the MD coordinators for providing with beam time and the operation team for setting up the cycles. Finally we would like to thank BLOND developers for their contributions to the simulation code.

[1] G. Apollinari, I. Béjar Alonso, O. Brüning, M. Lamont, and L. Rossi, CERN Technical Report No. CERN-2015005, 2015.

[2] A. Caldwell, E. Gschwendtner, K. Lotov, P. Muggli, and M. Wing (AWAKE Collaboration), Technical Reports No. CERN-SPSC-2013-013 and No. SPSC-TDR-003, 2013.

[3] H. Damerau, A. Funken, R. Garoby, S. Gilardoni, B. Goddard, K. Hanke, A. Lombardi, D. Manglunki, M. Meddahi, B. Mikulec, G. Rumolo, E. Shaposhnikova, M. Vretenar, and J. Coupard, CERN Technical Report No. CERN-ACC-2014-0337, 2014.

[4] T. Argyropoulos, in Proceedings of HB2014 (JACoW, East-Lansing, USA, 2014), THO4LR01, p. 404.

[5] A. Lasheen, T. Bohl, S. Hancock, E. Radvilas, T. Roggen, and E. Shaposhnikova, in Proc. of International Particle Accelerator Conference (IPAC'16), Busan, Korea, 2016 (JACoW, Geneva, Switzerland, 2016), p. 1670.

[6] A. Lasheen, CERN Technical Report No. CERN-ACCNOTE-2016-0074, 2016.

[7] J. E. Campelo, T. Argyropoulos, T. Bohl, F. Caspers, J. Esteban Müller, J. Ghini, A. Lasheen, D. Quartullo, B. Salvant, E. Shaposhnikova, and C. Zannini, in Proceedings of IPAC2015 (JACoW, Richmond, USA, 2015), MOPJE035, p. 360.

[8] J.E. Campelo, in Proceedings of IPAC2015 (JACoW, Richmond, VA, USA, 2015), MOPJE036, p. 363.

[9] T. Bohl, T.P. R. Linnecar, and E. Shaposhnikova, Measuring the Resonance Structure of Accelerator Impedance with Single Bunches, Phys. Rev. Lett. 78, 3109 (1997).

[10] T. Bohl, T.P. R. Linnecar, and E. Shaposhnikova, in Proceedings of EPAC 2002 (JACoW, Paris, France, 2002), WEPRI083, p. 1446.

[11] E. Shaposhnikova, in Proceedings of EPAC96 (JACoW, Barcelona, Spain, 1996), p. 1021.

[12] T. Bohl, CERN Technical Report No. Note-2006-37, 2006.

[13] T. Bohl, CERN Technical Report No. Note-2011-09, 2011.

[14] E. Shaposhnikova, T. Argyropoulos, T. Bohl, F. Caspers, A. Lasheen, J. Esteban Muller, B. Salvant, H. Timko, and J. E. Varela, in Proceedings of IPAC2014 (JACoW, Dresden, Germany, 2014), TUPME029, p. 1416. 
[15] CST MICROWAVE STUDIO, https://www.cst.com/ products/cstmws.

[16] B. W. Zotter and S. A. Kheifets, Impedances and Wakes in High-Energy Particle Accelerators (World Scientific, Singapore, 1998).

[17] H. Timko, J. E. Müller, A. Lasheen, and D. Quartullo, in Proc. of International Particle Accelerator Conference
(IPAC'16), Busan, Korea, May 8-13, 2016 (JACoW, Geneva, Switzerland, 2016), p. 3094.

[18] M. Migliorati, S. Persichelli, H. Damerau, S. Gilardoni, S. Hancock, and L. Palumbo, Beam-wall interaction in the CERN Proton Synchrotron for the LHC upgrade, Phys. Rev. ST Accel. Beams 16, 031001 (2013). 